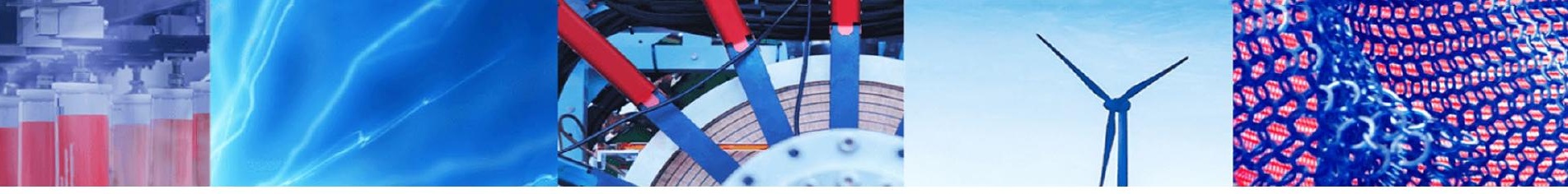

Research Article

\title{
Digital signatures over hash-entangled chains
}

\author{
Luis A. Lizama-Perez ${ }^{1}$ (1)
}

Received: 5 September 2019 / Accepted: 1 November 2019 / Published online: 7 November 2019

(c) Springer Nature Switzerland AG 2019

\begin{abstract}
In this work we introduce a new approach for digital signature based on hash chains. The method is supported on the well known security principles of hash functions. Some main advantages of this public key system include security and estimated efficiency through the so called signature chains. In addition, the algorithm is a promissory alternative for digital signatures for the quantum era.
\end{abstract}

Keywords Hash · Chain · Digital signature

\section{Introduction}

Generally speaking, a digital signature covers the methods useful to demonstrate irrevocably that a user has written a digital message. To achieve the goal, such algorithms have exploited some computational problems based on modular arithmetic or the security properties of hash functions. Unfortunately, methods based on the integer factorization problem or discrete logarithm fail under Shor's algorithm for the quantum era [1]. By contrast, hash functions are considered a good alternative to construct post-quantum algorithms [2]. In such scenario, we have developed a new method based on what we call hash-entangled chains. The term hash-entangled denotes that a hash-chain is linked to a previous chain. Later, we will give detail about the algorithm. First, in the next section, we will describe briefly some of the most relevant systems for digital signatures. Next, we will introduce the hash-entangled method to discuss, right away, the security issues and performance of the algorithm.

\subsection{Digital signatures}

Some methods have been developed to perform digital signatures. We can mention two main algorithms emerged among modular arithmetic which are based on the difficulty of the integer factorization problem: the RSA cryptosystem [3,4] and the Rabin cryptosystem [5]. Also, the Digital Signature Algorithm (DSA) $[4,6,7]$ is based on the difficulty of the discrete logarithm problem.

By contrast, other methods have been conceived over the basis of hash functions. For this case, we can mention the one time signature method (OTS) [8], OTS with Merkle Trees [9-11] and Lizama Certification Authority [12]. We identify some major advantages of hash functions which we summarize as follows:

- Well known security foundations

- A variety of algorithms is available

- A promissory alternative to be post-quantum

- Easy and fast to compute hash chains

- Specific hardware has been developed to compute hash chains

In the following section we will introduce a new method to achieve digital signatures based on hash functions. But before to introduce it, let us write the security assumptions of hash functions:

- Arbitrary data size input.

$\triangle$ Luis A. Lizama-Perez, luislizama@upp.edu.mx $\mid{ }^{1}$ Sección de Posgrado de la Universidad Politécnica de Pachuca, Ex-Hacienda de Santa Bárbara, 43830 Pachuca, Mexico. 
- Fixed data size output (e.g. 128, 160, 256 bits, etc).

- Feasible to compute. Is easy to compute $f(x)$ for all $x$.

- One way function. For any given hash value $h$ it is computationally infeasible to find $x$ given $f(x)=h$.

- Resistant to collision simple. For any given input $x$, it is computationally infeasible to find $y \neq x$ so that $f(y)=f(x)$.

- Resistant to strong collision. It is computationally infeasible to find any pair $(x, y)$ in such a way that $f(x)=f(y)$.

Before concluding this section we will mention that hash cryptography is resistant to quantum-crypto-analysis [13]. By contrast, methods based on modular arithmetic and the integer factoring problem or the discrete logarithm problem [14-16] are vulnerable to quantum computers capable to execute Peter Shor's algorithm [1]. However, a new approach based on quantum physics has emerged [17] which has been enhanced to resist quantum attacks $[18,19]$.

\section{Digital signatures using hash-chains}

For the present discussion, a hash chain is computed from a secret seed $s$ then performing a hash chain whose length is fixed to $2^{n}-1$ ( $n$ could be chosen between 128, 160, 256 , etc.). After applying the hash function over the seed it yields the head of the chain $f^{2^{n}-1}(s)$. If some middle point of the chain is shared or revealed, say $f^{2^{n}-1-x}(s)$, this value conducts to $f^{2^{n}-1}(s)$ by applying successively $x$ times the hash function, that is $f^{x}\left(f^{2^{n}-1-x}(s)\right)=f^{2^{n}-1}(s)$.

From the previous discussion, a message can be signed in the following manner: Alice publishes $f^{2^{n}-1}(s)$ where $s$ is a secret number and $n$ is publicly shared and it is fixed. Now, taking the hash of the message to be signed say $f(m)=h$ Alice publishes $m$ and $f^{2^{n}-1-h}(s)$. Any user who wants to verify the signature just compute $h$ and from here $f^{h}\left(f^{2^{n}-1-h}(s)\right)=f^{2^{n}-1}(s)$.

Unfortunately, the scheme described before is not completely functional at a practical level. First, consider that in the quantum era a secure hash function must be at least 256 bit length. Therefore, the length of the hash chain grows to $2^{256}-1$ which implies an enormous computational cost. Moreover, if we want to sign several messages with the same hash chain it must be enhanced properly. To overcome such limitations we will use multiple hash chains. In Fig. 1 we show the approach to reduce the length of the hash chain: the hash of the message $h_{0}=f\left(m_{0}\right)$ is divided in two portions $h_{00}$ and $h_{01}$ which correspond to the most and least significant bits of $h_{0}$. This reduces the length of the original hash chain to the half. As it can be inferred, increasing the number of hash chains also increases the number of the public head-chains to
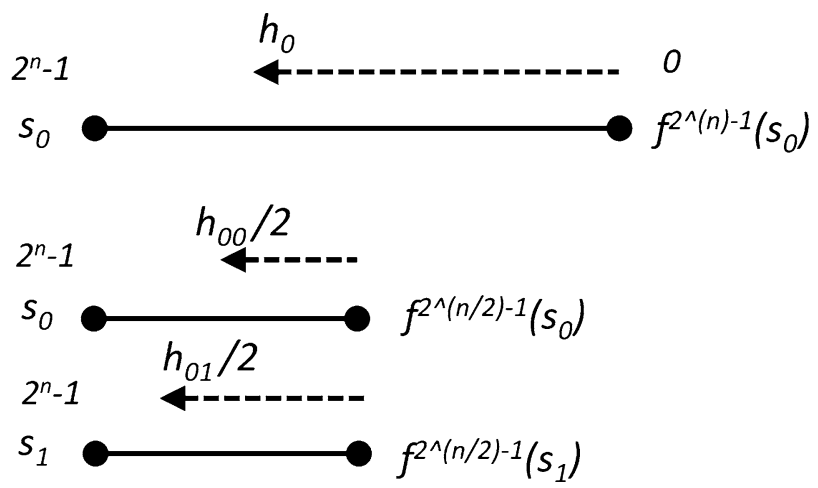

Fig. 1 The length of the hash chain could be reduced projecting the hash of the message over two hash chains

be stored (which constitutes the public key). Let $k$ be the number of the hash chains to be used. The effect of increasing $k$ will be discussed later.

\subsection{Digital signatures using multiple hash-chains}

Two hash chains could not be enough to minimize the computational effort required to sign a message. Now, we will describe the method using $k=4$ and a hash function of 128 bits, so any user (say Alice) must perform the following steps to sign messages (see the overall signature process in Fig. 2):

1. Alice chooses randomly four seeds $s_{1}, s_{2}, s_{3}$ and $s_{4}$, then she computes the hash-chains $f^{2^{n}-1}\left(s_{1}\right)$, $f^{2^{n}-1}\left(s_{2}\right), f^{2^{n}-1}\left(s_{3}\right)$ and $f^{2^{n}-1}\left(s_{4}\right)$. For this example, the size of each seed is $\left|s_{i}\right|=128$ bits. The number $n$ is fixed to 128 / $k$ thus the length of the hash chain is $2^{32}-1$. Now, Alice publishes her public head-chains: $P_{u_{A}}=\left\{f^{2^{32}-1}\left(s_{1}\right), f^{2^{32}-1}\left(s_{2}\right), f^{3^{32}-1}\left(s_{3}\right), f^{2^{32}-1}\left(s_{4}\right)\right\}$.

2. To sign the first message $m_{1}$, Alice computes the hash of the message which is denoted as $h_{1}=f\left(m_{1}\right)$. Then, she separates the 128 bits in tree portions of 32 bits. Let us denote such portions as $h_{1}{ }^{00}, h_{1}{ }^{01}, h_{1}{ }^{10}$ and $h_{1}{ }^{11}$. Alice takes the seeds and computes $f^{2^{32}-1-h_{1}{ }^{00}}\left(s_{1}\right)$, $f^{2^{32}-1-h_{1}{ }^{01}}\left(s_{2}\right), f^{2^{32}-1-h_{1}{ }^{10}}\left(s_{3}\right)$ and $f^{2^{32}-1-h_{1}{ }^{11}}\left(s_{4}\right)$.

3. Alice publishes the message $m_{1}$, so any user who wants to verify the signature must check that: $f^{h_{1}{ }^{00}}\left(f^{2^{32}-1-h_{1}{ }^{00}}\left(s_{1}\right)\right)=f^{2^{32}-1}\left(s_{1}\right), f^{h_{1}{ }^{01}}\left(f^{32}-1-h_{1}{ }^{01}\left(s_{2}\right)\right)=f^{3^{32}-1}\left(s_{2}\right)$,

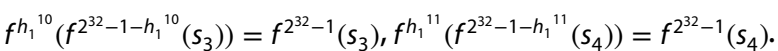

4. Finally, Alice generates and publishes the next head chains $f^{2^{32}-1}\left(s_{5}\right)$ and $f^{2^{32}-1}\left(s_{6}\right), f^{2^{32}-1}\left(s_{7}\right), f^{2^{32}-1}\left(s_{8}\right)$ as they can be seen in Fig. 2. The process continues to sign an undefined number of incoming messages. 
Fig. 2 The overall signature publication process with $k=4$. Alice sends to the receiver the corresponding messages $m_{i}$. The super-indices $00,01,10$ and 11 denote the portion bits of the hash code. Alice continues signing an undefined number of messages
Timeline of Alice's signature process

$$
\begin{aligned}
& \leftarrow f^{2^{n}-1}\left(s_{1}\right), f^{2^{n}-1}\left(s_{2}\right), f^{2^{n}-1}\left(s_{3}\right), f^{2^{n}-1}\left(s_{4}\right) \\
& \downarrow \leftarrow m_{1}, f^{2^{n}-1-h_{1}{ }^{00}}\left(s_{1}\right), f^{2^{n}-1-h_{1}{ }^{01}}\left(s_{2}\right), f^{2^{n}-1-h_{1}{ }^{10}}\left(s_{3}\right), f^{2^{n}-1-h_{1}{ }^{11}}\left(s_{3}\right) \\
& f^{2^{n}-1}\left(s_{5}\right), f^{2^{n}-1}\left(s_{6}\right), f^{2^{n}-1}\left(s_{7}\right), f^{2^{n}-1}\left(s_{8}\right) \\
& \downarrow \leftarrow m_{2}, f^{2^{n}-1-h_{2}{ }^{00}}\left(s_{5}\right), f^{2^{n}-1-h_{2}{ }^{01}}\left(s_{6}\right), f^{2^{n}-1-h_{2}{ }^{10}}\left(s_{7}\right), f^{2^{n}-1-h_{2}{ }^{11}}\left(s_{8}\right) \\
& f^{2^{n}-1}\left(s_{9}\right), f^{2^{n}-1}\left(s_{10}\right), f^{2^{n}-1}\left(s_{11}\right), f^{2^{n}-1}\left(s_{12}\right) \\
& \downarrow m_{i}, f^{2^{n}-1-h_{i}{ }^{00}}\left(s_{j}\right), f^{2^{n}-1-h_{i}{ }^{01}}\left(s_{j+1}\right), f^{2^{n}-1-h_{i}{ }^{10}}\left(s_{j+2}\right), f^{2^{n}-1-h_{i}{ }^{11}}\left(s_{j+3}\right) \\
& f^{2^{n}-1}\left(s_{j+4}\right), f^{2^{n}-1}\left(s_{j+5}\right), f^{2^{n}-1}\left(s_{j+6}\right), f^{2^{n}-1}\left(s_{j+7}\right)
\end{aligned}
$$
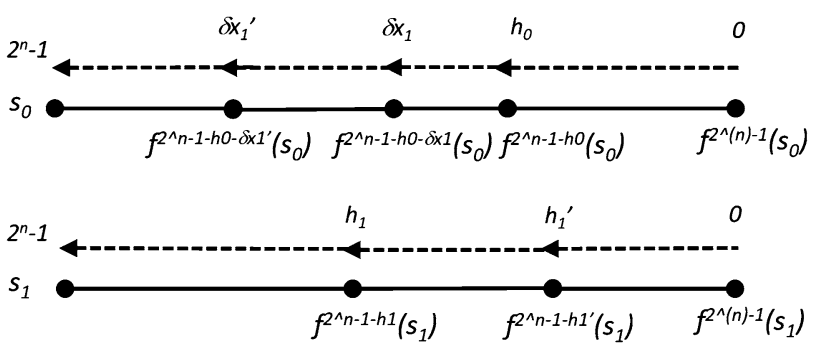

Fig. 3 Eve cannot compute $f^{2^{n}-1-h_{0}-\delta x_{1}^{\prime}}\left(s_{0}\right)$ because this operation requires to reverse the hash function from $f^{2^{n}-1-h_{0}-\delta x_{1}}\left(s_{0}\right)$ to $f^{2^{n}-1-h_{0}-\delta x_{1}^{\prime}}\left(s_{0}\right)$

\section{The entanglement algorithm}

The security of the method is based in what we call the entanglement algorithm. To describe it, let us consider firstly the case $k=1$. At the end of this section, we will generalize the algorithm.

Suppose Alice has signed a message $m_{0}$ using $f^{2^{n}-1}\left(s_{0}\right)$, it means she has revealed $f^{2^{n}-1-h_{0}}\left(s_{0}\right)$ where $h_{0}=f\left(m_{0}\right)$. If Alice signs a new message $m_{1}$ so $h_{1}=f\left(m_{1}\right)$ an intruder could fake the signature of $m_{1}^{\prime}$ provided $h_{1}^{\prime}<h_{1}$ where $h_{1}^{\prime}=f\left(m_{1}^{\prime}\right)$ because it is easy to compute the hash chain in such direction (see bottom of Fig. 3).

To surpass such weakness we propose the entanglement algorithm which is based on the fact that in a hash chain after signing a message always remains unused some points at the left of the computed values of the chain. This is so because $2^{n}-1$ is the maximum number that could be allocated in the chain so $2^{n}-1>h_{i}$ (see Fig. 3).

The first step is compute the relation between the complement of $h_{1}$ that is $2^{n}-1-h_{1}$ over the length of the hash chain $2^{n}-1$ then projecting it over $2^{n}-1-h_{0}$ as indicated in Eq. 1. We must demonstrate that in the presence of an attacker who is trying to fake $h_{1}$ with $h_{1^{\prime}}^{\prime}$ the point that defines $\frac{2^{n}-1-h_{1}^{\prime}}{2^{n}-1}\left(2^{n}-1-h_{0}\right)$ is always at the left of $\frac{2^{n}-1-h_{1}}{2^{n}-1}\left(2^{n}-1-h_{0}\right)$ in the hash chain of $s_{0}$ (see Fig. 3). Such condition is represented in Eq. 2. We must remark that $\delta x_{1}$ defines a unique point inside the hash chain according to hash principles (see Fig. 3 ). The steps indicated after Eq. 2 allow us to conclude that $h_{1}^{\prime}<h_{1}$ which is always true as it was commented previously.

We introduce the chain ratio $\delta x_{1}$ in Eq. 3 that is computed as $h_{1}-\frac{h_{0} h_{1}}{2^{n}-1}$ (a rounding function must be applied to $\delta x_{1}$ if it were necessary), so any user who wants to verify the signature must apply Eq. 4 . To be clearer, let us enumerate again the steps of the signature process:

1. Assume Alice has previously published $f^{2^{n}-1}\left(s_{1}\right)$. To sign the message $m_{1}$ Alice computes the chain ratio $\delta x_{1}$ to obtain $f^{2^{n}-1-h_{0}-\delta x_{1}}\left(s_{0}\right)$, then she publishes it along $m_{1}$.

2. Using $m_{0}$ and $m_{1}$ Bob computes $\delta x_{1}$ and verifies that: 
- $f^{\delta x_{1}}\left(f^{2^{n}-1-h_{0}-\delta x_{1}}\left(s_{0}\right)\right)=f^{2^{n}-1-h_{0}}\left(s_{0}\right) \quad$ (remember that $f^{2^{n}-1-h_{0}}\left(s_{0}\right)$ has been previously published by Alice.

- $f^{h_{1}}\left(f^{2^{n}-1-h_{1}}\left(s_{1}\right)\right)=f^{2^{n}-1}\left(s_{1}\right)$

$\frac{2^{n}-1-h_{1}}{2^{n}-1}\left(2^{n}-1-h_{0}\right)$

$\left(1-\frac{h_{1}}{2^{n}-1}\right)\left(2^{n}-1-h_{0}\right)=2^{n}-1-h_{0}-h_{1}+\frac{h_{0} h_{1}}{2^{n}-1}$

$2^{n}-1-h_{0}-h_{1}^{\prime}+\frac{h_{0} h_{1}^{\prime}}{2^{n}-1}>2^{n}-1-h_{0}-h_{1}+\frac{h_{0} h_{1}}{2^{n}-1}$

$-h_{1}^{\prime}+\frac{h_{0} h_{1}^{\prime}}{2^{n}-1}>-h_{1}+\frac{h_{0} h_{1}}{2^{n}-1}$

$h_{1}^{\prime}-\frac{h_{0} h_{1}^{\prime}}{2^{n}-1}<h_{1}-\frac{h_{0} h_{1}}{2^{n}-1}$

$h_{1}^{\prime}\left(1-\frac{h_{0}}{2^{n}-1}\right)<h_{1}\left(1-\frac{h_{0}}{2^{n}-1}\right)$

$h_{1}^{\prime}<h_{1}$

$\delta x_{1}=h_{1}-\frac{h_{0} h_{1}}{2^{n}-1}$

$f^{\delta x_{1}}\left(f^{2^{n}-1-h_{0}-\delta x_{1}}\left(s_{0}\right)\right)=f^{2^{n}-1-h_{0}}\left(s_{0}\right)$

An intruder who intercepts $T_{1}$ cannot forward to Bob another message say $T_{1}^{\prime}$ signed as $T_{1}$, because:

- As stated before, if $h_{1}^{\prime}>h_{1}$, Eve would try to forge the message $m_{1}^{\prime}$ but this condition violates Eq. 2 . This requires Eve to compute the hash function from $f^{2^{n}-1-h_{0}-\delta x_{1}}\left(s_{0}\right)$ to $f^{2^{n}-1-h_{0}-\delta x_{1}^{\prime}}\left(s_{0}\right)$ (see Fig. 3). This operation implies reversing (several times) the hash function which it is assumed to be computationally infeasible.

The strategy described before can be generalized to multiple hash chains. For example, when $k=4$ (Alice signs the first message $h_{0}$ using $s_{k}$, $k=0, \ldots, 3)$. To sign the next message $h_{1}$, Alice publishes $f^{2^{n}-1-h_{1}-\delta x_{k}}\left(s_{k}, k=4, \ldots, 7\right.$. Bob must compute $\delta x_{k}$ and verify that $f^{\delta x_{k}}\left(f^{2^{n}-1-h_{1}-\delta x_{k}}\left(s_{k}\right)\right)=f^{2^{n}-1-h_{1}}\left(s_{k}\right)$, $k=4, \ldots, 7$ and so on. It must be clear that the computation process of $f^{\delta x_{k}}\left(f^{2^{n}-1-h_{1}-\delta x_{k}}\left(s_{k}\right)\right)$ is much faster than $f^{h_{1}}\left(f^{2^{n}-1-h_{1}}\left(s_{k}\right)\right)=f^{2^{n}-1}\left(s_{k}\right)$ because $\delta x_{1}=h_{1}-\frac{h_{0} h_{1}}{2^{n}-1}$ is always small compared with $h_{1}$ (the maximum value for $h_{0}$ and $h_{1}$ is $2^{n}-1$ ) (Fig. 4).
Timeline of Alice's signature process

$$
\begin{aligned}
& \downarrow f^{2^{n}-1}\left(s_{0}\right) \\
& \downarrow \leftarrow m_{0}, f^{2^{n}-1-h_{0}}\left(s_{0}\right) \\
& \leftarrow f^{2^{n}-1}\left(s_{1}\right) \\
& \downarrow m_{1}, f^{2^{n}-1-h_{1}}\left(s_{1}\right), f^{2^{n}-1-h_{0}-\delta x_{1}}\left(s_{0}\right) \\
& f^{2^{n}-1}\left(s_{2}\right) \\
& \downarrow \leftarrow m_{2}, f^{2^{n}-1-h_{2}}\left(s_{2}\right), f^{2^{n}-1-h_{1}-\delta x_{2}}\left(s_{1}\right) \\
& f^{2^{n}-1}\left(s_{i}\right) \\
& \downarrow \leftarrow m_{i}, f^{2^{n}-1-h_{i}}\left(s_{i}\right), f^{2^{n}-1-h_{i-1}-\delta x_{i}}\left(s_{i-1}\right)
\end{aligned}
$$

Fig. 4 The signature publication process with $\delta x$ security. Alice sends to the receiver the corresponding message $m_{i}$

\subsection{Single-chain}

Suppose Alice and Bob have recorded its signature history in a web service where both of them have been registered. In this context, we call single-chain to this user-chain history. Now, Bob needs to verify Alice's single-chain who wants to perform a digital transaction with him. To achieve this goal Bob must perform the next steps:

- Firstly, Bob must verify the hash of the first two messages signed by Alice $h_{0}=f\left(m_{0}\right)$ and $h_{1}=f\left(m_{1}\right)$.

- Then he computes $\delta x_{1}=h_{1}-\frac{h_{0} h_{1}}{2^{n}-1}$.

- Using $f^{2^{n}-1-h_{1}-\delta x_{1}}\left(s_{0}\right)$ Bob verifies that $f^{\delta x_{1}}\left(f^{2^{n}-1-h_{1}-\delta x_{1}}\left(s_{0}\right)\right)=f^{2^{n}-1-h_{1}}\left(s_{0}\right)$.

- Bob obtains the hash of the next message $h_{2}=f\left(m_{2}\right)$.

- Using $f^{2^{n}-1-h_{2}-\delta x_{2}}\left(s_{1}\right)$ Bob verifies that $f^{\delta x_{2}}\left(f^{2^{n}-1-h_{2}-\delta x_{2}}\left(s_{1}\right)\right)=f^{2^{n}-1-h_{2}}\left(s_{1}\right)$.

- The verification process continues until Bob reaches the current signature executed by Alice.

The method described before prevents Bob to verify each Alice's digital signature of each chain through the computation $f^{h_{j}}\left(f^{2^{n}-1-h_{j}}\left(s_{j}\right)\right)=f^{2^{n}-1}\left(s_{j}\right)$ which requires a larger verification process. It is supported by the fact that in the 


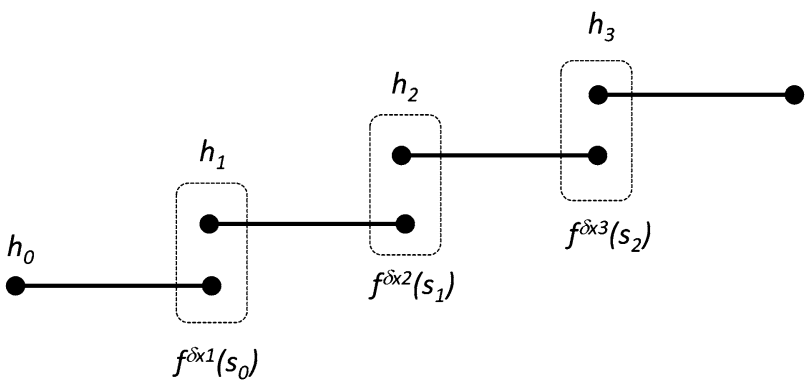

Fig. 5 Alice verifies $f^{\delta x_{j}}\left(f^{2^{n}-1-h_{j}-\delta x_{j}}\left(s_{j-1}\right)\right)$ instead of $f^{h_{j}}\left(f^{2^{n}-1-h_{j}}\left(s_{j}\right)\right)$ which requires a larger hash chain computation

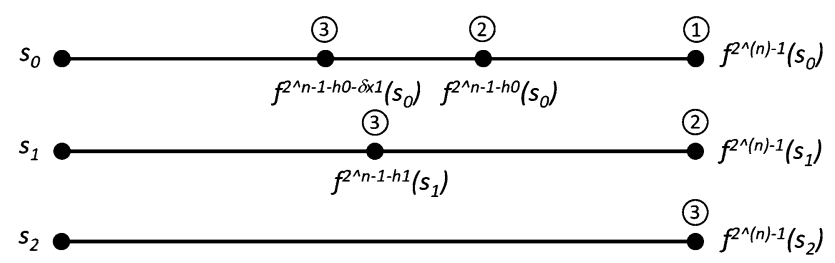

Fig. 6 The order at which the information is published in the web service. In step 2 , the web service substitutes $f^{2^{n}-1}\left(s_{0}\right)$ by $f^{2^{n}-1-h_{0}}\left(s_{0}\right)$

relation $\delta x_{j}=h_{j}-\frac{h_{j-1} h_{j}}{2^{n}-1}, \delta x_{j}$ is derived from two values $h_{j}$ and $h_{j-1}$. Thus, given $h_{j}$ and $h_{j-1}$ they produce a unique hash-sequence of the messages that have been previously signed by the user. We write it symbolically in Eq. 5 and it is represented in Fig. 5:

$\delta x_{1} \rightarrow h_{0} h_{1}, \delta x_{2} \rightarrow h_{1} h_{2} \ldots \delta x_{k} \rightarrow h_{k-1} h_{k} \ldots \delta x_{n} \rightarrow h_{n-1} h_{n}$

Before analyzing the performance analysis in detail, we can introduce the following optimization of the storing space. The web service would store the hash of the message, the public-head hash $f^{2^{n}-1}\left(s_{i}\right)$, the signing hash $f^{2^{n}-1-h_{i}}\left(s_{i}\right)$ and the security hash $f^{2^{n}-1-h_{i}-\delta x_{i+1}}\left(s_{i}\right)$ :

1. $h_{i}$

2. $f^{2^{n}-1}\left(s_{i}\right)$
3. $f^{2^{n}-1-h_{i}}\left(s_{i}\right)$

4. $f^{2^{n}-1-h_{i}-\delta x_{i+1}}\left(s_{i}\right)$

However, after a new message $h_{i}$ is verified, then $f^{2^{n}-1-h_{i}}\left(s_{i}\right)$ could take the place of $f^{2^{n}-1}\left(s_{i}\right)$ to be stored in the web service, so we can keep just three hashes: the hash of the message, the signing hash and the security hash (see Fig. 6).

1. $h_{i}$

2. $f^{2^{n}-1-h_{i}}\left(s_{i}\right)$

3. $f^{2^{n}-1-h_{i}-\delta x_{i+1}}\left(s_{i}\right)$

To avoid an attack over $f^{2^{n}-1-h_{i}}\left(s_{i}\right)$ the hash of the signing message $h_{i}$ is computed applying the hash function to the concatenation of the hash of the current message $m_{i}$ and the hash of the previous one $h_{i-1}$. Symbolically, it is written in Eq. 6.

$h_{0}=f\left(m_{0}\right), h_{1}=f\left(h_{0} \| f\left(m_{1}\right)\right)$,

$h_{2}=f\left(h_{1} \| f\left(m_{2}\right)\right), \ldots h_{i}=f\left(h_{i-1} \| f\left(m_{i}\right)\right)$

\section{Performance analysis}

As discussed before, using a 256-bit hash requires to compute the hash chain as $f^{2^{256}-1}(s)$ which could be and extremely heavy process. However, as commented earlier the hash of the message can be divided into $k$ chains.

We will analyze the performance of the signature method for some $k$ values at a hash rate of 15,840,454 hash/s according to the data published on the site bitcointalk.org. Let us call $N$ to the size of the hash function used in the signature process. So we have $N=\sum_{i=1}^{k} \cdot n_{i}$. For example, if $N=128$ and $k=4$ they are generated four chains of 64 bits.

Tables 1, 2 and 3 show the size of the keys and the estimated time required to compute the hash chains for $k=\{2,4,8,16,32\}$ and $N=\{128,160,256\}$ at a hash speed of $10^{7}$ hash/s. Finally, Table 4 summarize the best results in the current state of computer technology.
Table 1 The hash length is $N=256$

\begin{tabular}{llll}
\hline$k: f^{2^{n}-1}\left(s_{i}\right)$ where $i=1, \ldots, k$ & $k \cdot 256$ (bits) & Number of hashes & Required time $(s)$ \\
\hline $2: f^{|128|}\left(s_{1}\right), f^{|128|}\left(s_{2}\right)$ & 512 & $2^{128}-10^{38}$ & $\sim 10^{31}$ \\
$4: f^{|64|}\left(s_{1}\right), f^{|64|}\left(s_{2}\right), \ldots f^{|64|}\left(s_{4}\right)$ & 1024 & $2^{64}-10^{18}$ & $\sim 10^{11}$ \\
$8: f^{|32|}\left(s_{1}\right), f^{|32|}\left(s_{2}\right), \ldots f^{|32|}\left(s_{8}\right)$ & 2048 & $2^{32}-10^{8}$ & $\sim 10^{1}$ \\
$16: f^{|16|}\left(s_{1}\right), f^{|16|}\left(s_{2}\right), \ldots f^{|16|}\left(s_{16}\right)$ & 4096 & $2^{16}-10^{4}$ & $\sim 10^{-3}$ \\
$32: f^{|8|}\left(s_{1}\right), f^{|8|}\left(s_{2}\right), \ldots f^{|8|}\left(s_{32}\right)$ & 8192 & $2^{8}-10^{1}$ & $\sim 10^{-6}$ \\
\hline
\end{tabular}

It has been assumed a hash speed of $10^{7}$ hash/s. The notation $f^{|e|}\left(s_{i}\right)$ means that the exponent $|e|$ is computed as $2^{e}$. The size of the key is obtained as $k \cdot N$. The time has been derived according to the site bitcointalk.org with a processor AMD Phenom(tm) II X6 1055T, 6 cores, 2819.765 MHz, 15,840,454 hash/s 
Table $2 N=160$ bits at a hash speed of $10^{7}$ hash/s

\begin{tabular}{lcll}
\hline$k: f^{2^{n}-1}\left(s_{i}\right)$ where $i=1, \ldots, k$ & $k \cdot 160($ bits) & Number of hashes & Required time $(s)$ \\
\hline $2: f^{|80|}\left(s_{1}\right), f^{|80|}\left(s_{2}\right)$ & 320 & $2^{80}-10^{23}$ & $\sim 10^{16}$ \\
$4: f^{|40|}\left(s_{1}\right), f^{|40|}\left(s_{2}\right), \ldots f^{|40|}\left(s_{4}\right)$ & 640 & $2^{40}-10^{11}$ & $\sim 10^{4}$ \\
$8: f^{|20|}\left(s_{1}\right), f^{|20|}\left(s_{2}\right), \ldots f^{|10|}\left(s_{8}\right)$ & 1280 & $2^{20}-10^{6}$ & $\sim 10^{-1}$ \\
$16: f^{|10|}\left(s_{1}\right), f^{|10|}\left(s_{2}\right), \ldots f^{|5|}\left(s_{16}\right)$ & 2560 & $2^{10}-10^{2}$ & $\sim 10^{-5}$ \\
\hline
\end{tabular}

\begin{tabular}{lcll}
\hline$k: f^{2^{n}-1}\left(s_{i}\right)$ where $i=1, \ldots, k$ (bits) & $k \cdot 128$ & Number of hashes & Required time $(s)$ \\
\hline $2: f^{|64|}\left(s_{1}\right), f^{|64|}\left(s_{2}\right)$ & 256 & $2^{64}-10^{18}$ & $\sim 10^{11}$ \\
$4: f^{|32|}\left(s_{1}\right), f^{|32|}\left(s_{2}\right), \ldots f^{|32|}\left(s_{4}\right)$ & 512 & $2^{32}-10^{8}$ & $\sim 10^{1}$ \\
$8: f^{|16|}\left(s_{1}\right), f^{|16|}\left(s_{2}\right), \ldots f^{|16|}\left(s_{8}\right)$ & 1024 & $2^{16}-10^{4}$ & $\sim 10^{-3}$ \\
$16: f^{|8|}\left(s_{1}\right), f^{|8|}\left(s_{2}\right), \ldots f^{|8|}\left(s_{16}\right)$ & 2048 & $2^{8}-10^{1}$ & $\sim 10^{-6}$ \\
$32: f^{|4|}\left(s_{1}\right), f^{|4|}\left(s_{2}\right), \ldots f^{|4|}\left(s_{32}\right)$ & 4096 & $2^{4}-10^{0}$ & $\sim 10^{-7}$ \\
\hline
\end{tabular}

Table $3 N=128$ bits at a hash speed of $10^{7}$ hash/s
Table 4 Some promising results are 128 bit-hash and 1024 bits key-size, 160 bit-hash with 1280 key-bit and 256 bithash with 4096 key-bit

\begin{tabular}{lllll}
\hline$k: f^{2^{n}-1}\left(s_{i}\right)$ where $i=1, \ldots, k$ & $\begin{array}{l}\text { Hash func- } \\
\text { tion (bits) }\end{array}$ & Key size (bits) & Number of hashes & Required time $(s)$ \\
\hline $8: f^{|16|}\left(s_{1}\right), f^{|16|}\left(s_{2}\right), \ldots f^{|16|}\left(s_{8}\right)$ & 128 & 1024 & $2^{16}-10^{4}$ & $\sim 10^{-3}$ \\
$8: f^{|20|}\left(s_{1}\right), f^{|20|}\left(s_{2}\right), \ldots f^{|20|}\left(s_{8}\right)$ & 160 & 1280 & $2^{20}-10^{6}$ & $\sim 10^{-1}$ \\
$8: f^{|32|}\left(s_{1}\right), f^{|32|}\left(s_{2}\right), \ldots f^{|32|}\left(s_{8}\right)$ & 256 & 2048 & $2^{32}-10^{8}$ & $\sim 10^{1}$ \\
$16: f^{|16|}\left(s_{1}\right), f^{|16|}\left(s_{2}\right), \ldots f^{|16|}\left(s_{16}\right)$ & 256 & 4096 & $2^{16}-10^{4}$ & $\sim 10^{-3}$ \\
\hline
\end{tabular}

The private key is formed by the seeds Alice uses to compute the chains. For example, for $k=16$ we have the following seeds: $\left\{s_{0}, s_{1}, \ldots, s_{15}\right\}$. A convenient method to reduce the size of the private key is represented in Eq. 7 .

$s_{0}, s_{1}, s_{2}=f\left(s_{0} \| s_{1}\right), s_{3}=f\left(s_{1} \| s_{2}\right), \ldots$

The size of the private key is reduced to the length of two hashes. If we are using SHA-256, then the size of the private key (to be stored in a mobile device) is fixed to 512 bits.

\subsection{System implementation}

Figure 7 shows the system architecture of the signature system. For this implementation we used SHA-256 and $k=6$. The signature file occupies 32 hashes (8192 bits), which can be viewed according to the points in Fig. 6:

- 16 points marked with the number 3 in the chain starting with $s_{0}$.

- 16 verification points marked with the number 3 in the chain of $s_{1}$.

The Web file contains 32 hashes ( 8192 bits) plus the hash of the message:

SN Applied Sciences
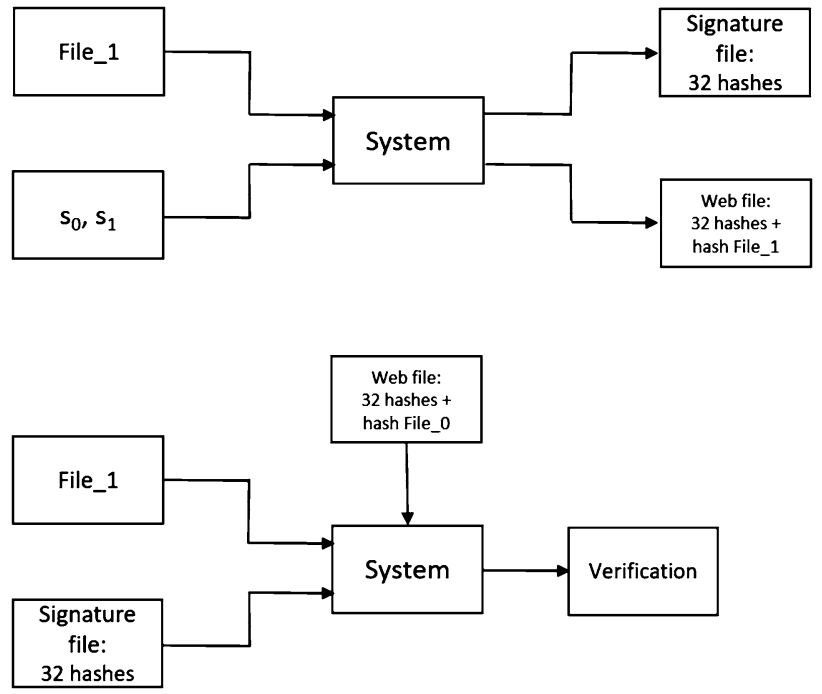

Fig. 7 Digital system architecture for signing a file (upper) and the signature verification (low)

- 16 new head-chains, points marked with the number 3 in the chain of $s_{2}$.

- 16 hashes, points marked with the number 2 in the chain of $s_{0}$ used by the web system to replace the headchains (marked with the number 1 in the chain of $s_{0}$ in Fig. 6). 
Table 5 Comparative analysis between some of the main blockchain-based protocols

\begin{tabular}{lllllll}
\hline Protocol & Data base & Signature (s) & Verification of the chain & Block size & Total size & Q \\
\hline Bitcoin & \multirow{2}{*}{ Blockchain } & $10^{-3}$ & Minutes to hours & $\sim \mathrm{MB}$ & $242 \mathrm{~GB}$ & No \\
Ethereum & & $10^{-3}$ & $10-20 \mathrm{~s}$ & $\sim \mathrm{kB}$ & $600 \mathrm{~GB}$ & No \\
\multirow{2}{*}{ Single-chain } & \multirow{2}{*}{ Distributed } & $1.6-2.6$ & $1-2 \mathrm{~min}$ & $\sim \mathrm{kB}$ & - & Yes \\
\hline
\end{tabular}

The distributed data base of the single-chain protocol means that exists a data base by each user. The column $\mathrm{Q}$, points out if the protocol survives in the quantum era
- The hash of the message written as hash File_1 in Fig. 7.

The user stores the seeds $s_{0}$ and $s_{1}$ (possibly in his mobile device) which are used by the signature system to generate the next seeds and the head-chains (public key). In this implementation, the time to generate the public headchains goes from 1.1 to $1.3 \mathrm{~s}$. Alice sends the file of the message (File_1) to Bob accompanied by its signature file. After Bob downloads the Web file, the signature verification is achieved successfully provided (see Fig. 6):

- He can go from the (16) points marked with the number 3 to the (16) points marked with the number 2 in the chain of $s_{1}$.

- Bob can move from the (16) points marked with the number 3 to the (16) points marked with the number 2 in the chain of $s_{0}$.

The verification of the signature file takes around $0.3 \mathrm{~s}$ in our implementation. However, verification of the singlechain varies according to its length, occupying few minutes to complete the verification.

\subsection{Applications}

Digital signatures based on hash chains, as the ones compared in Table 5, have a wide range of applications because transactions are highly traceable and verified before they are stored in an encrypted data base. Although exist some interesting applications for this kind of service one of the most relevant is the electronic payment service. Other potential uses include smart contracts, tracing physical or digital assets in the scope of the Internet of Things (IOT). Moreover, it could be properly enhanced other services as healthcare, music, government and identity.

To reduce the time to verify the single-chain of each user, it could be defined middle certificated points. It could be explored in a future implementation work.

\section{Conclusions}

Signature methods developed so far using hash functions are either ephemeral, as Lamport signature or they are not completely scalable as Merkle trees or Lizama hash signature. In this work, we have introduced a new approach based on hash functions and hash chains, which is capable to work over a public key infrastructure so that users can initialize remotely their hash-chain in its own computational device.

The signature method, which can exploited as a single block chain is based on the security principles of hash functions and our analyses suggests that the signature chains introduced here are scalable with today's hash technology. The method does not require other math function apart of hash function, therefore the algorithm is a promissory alternative for digital signatures in the quantum era.

\section{Compliance with ethical standards}

Conflict of interest The author declares that they have no competing interests.

\section{References}

1. Shor PW (1994) Algorithms for quantum computation: discrete logarithms and factoring. In: 35th Annual Symposium on Foundations of Computer Science, 1994 proceedings. IEEE, pp 124-134

2. Buchmann JA, Butin D, Göpfert F, Petzoldt A (2016) Post-quantum cryptography: state of the art. In: Ryan P, Naccache D, Quisquater JJ (eds) The new codebreakers. Springer, Berlin, pp 88-108

3. Rivest RL, Shamir A, Adleman L (1978) A method for obtaining digital signatures and public-key cryptosystems. Commun ACM 21(2):120-126

4. FIPS P (2009) 186-3. Digital signature standard (DSS) 
5. Rabin MO (1979) Digitalized signatures and public-key functions as intractable as factorization. Tech. rep., Massachusetts Inst of Tech Cambridge Lab for Computer Science

6. FIPS N (1998) 186-1. Digital Signature Standard

7. FIPS P (2000) 186-2. Digital signature standard (dss). National Institute of Standards and Technology (NIST)

8. Lamport L (1979) Constructing digital signatures from a oneway function. Tech. rep., Technical Report CSL-98, SRI International Palo Alto

9. Merkle RC (1982) Method of providing digital signatures. US Patent 4,309,569

10. Merkle RC (1987) A digital signature based on a conventional encryption function. In: Advances in cryptology, CRYPTO 87. Springer, pp 369-378

11. Merkle RC (1989) A certified digital signature. In: Advances in cryptology, CRYPTO 89 proceedings. Springer, pp 218-238

12. Adrián LPL, Javier MAL, Seleyda HMF, Adrián LSL, Eric S-A (2019) Public hash signature for mobile network devices. Ing Investig Tecnol 20(2):1-10

13. Perlner RA, Cooper DA (2009) Quantum resistant public key cryptography: a survey. In: Proceedings of the 8th symposium on identity and trust on the internet. ACM, pp 85-93
14. Nielsen MA, Chuang IL (2010) Quantum computation and quantum information. Cambridge University Press, Cambridge

15. Dattani NS, Bryans N (2014) Quantum factorization of 56153 with only 4 qubits. arXiv preprint arXiv:1411.6758

16. Dridi R, Alghassi H (2016) Prime factorization using quantum annealing and computational algebraic geometry. arXiv preprint arXiv:1604.05796

17. Bennett $\mathrm{CH}$, Brassard G (1984) Quantum cryptography: public key distribution and coin tossing. In: Int. conf. on computers, systems and signal processing, Bangalore, pp 175-179

18. Lizama-Pérez LA, López JM, De Carlos-López E, Venegas-Andraca SE (2014) Quantum flows for secret key distribution in the presence of the photon number splitting attack. Entropy 16(6):3121-3135

19. Lizama-Pérez LA, López JM, De Carlos López E (2016) Quantum key distribution in the presence of the intercept-resend with faked states attack. Entropy 19(1):4

Publisher's Note Springer Nature remains neutral with regard to jurisdictional claims in published maps and institutional affiliations. 